\title{
Effects of alprostadil combined with tanshinone Ila injection on microcirculation disorder, outcomes, and cardiac function in AMI patients after $\mathrm{PCI}$
}

\author{
Yang Lu, Yongjin Yan, Xiaofeng Liu \\ Department of Cardiovascular Medicine, Affiliated Haian Hospital of Nantong University, Nantong, China \\ Contributions: (I) Conception and design: Y Lu; (II) Administrative support: Y Yan; (III) Provision of study materials or patients: X Liu; (IV) \\ Collection and assembly of data: Y Lu; (V) Data analysis and interpretation: Y Lu; (VI) Manuscript writing: All authors; (VII) Final approval of \\ manuscript: All authors. \\ Correspondence to: Yang Lu. Department of Cardiovascular Medicine, Affiliated Haian Hospital of Nantong University, Nantong, China. \\ Email: 1624359154@qq.com.
}

Background Alprostadil can effectively dilate blood vessels, improve cardiac microcirculation, and reduce
cardiac load. Tanshinone IIa injection can protect against atherosclerosis and reduce myocardial oxygen
consumption. However, the effects of alprostadil combined with tanshinone IIa injection on microcirculation
disorder, outcomes, and cardiac function in patients with acute myocardial infarction (AMI) after
percutaneous coronary intervention (PCI) are still not fully clear.

Methods: A total of 300 AMI patients who underwent PCI in our hospital from January 2013 to June 2018 were randomly selected and divided into group A, B, C by using the random number table method, with 100 patients in each group. The group A was treated with alprostadil, the group B was treated with tanshinone IIa injection, and the group C was treated with alprostadil combined with tanshinone IIa injection. 7 days after treatment, the cardiac functions of all patients were observed by ultrasonic Doppler, as were the microcirculations by myocardial contrast echocardiography (MCE). The major adverse cardiac events (MACEs) in both groups were observed in the 12-month follow-up.

Results: After treatment, the left ventricular end-diastolic diameter (LVEDD), end-diastolic left ventricular posterior wall thickness (LVPWD), left ventricular ejection fraction (LVEF), left ventricular end-systolic diameter (LVESD), interventricular septum thickness (IVST), and ratio of maximal early to late diastolic filling velocities (E/A) in the group $\mathrm{C}$ were superior to those in the group $\mathrm{A}$ and $\mathrm{B}$, the differences were statistically significant $(\mathrm{P}<0.05)$. After treatment, $\mathrm{MCE}$ showed that the $\mathrm{A} \beta$ value of the group aC was higher than that of the group $\mathrm{A}$ and $\mathrm{B}$, the difference was statistically significant $(\mathrm{P}<0.05)$. The thrombolysis in myocardial infarction myocardial perfusion grade classification showed that the patients with grades $2-3$ were more abundant in the group $\mathrm{C}$ than the group $\mathrm{A}$ and $\mathrm{B}$, the difference was statistically significant $(\mathrm{P}<0.05)$. The incidences of MACEs, such as malignant arrhythmia, recurrent heart failure (HF), recurrent myocardial infarction, and death, in the group $\mathrm{C}$ were significantly lower than those in the group $\mathrm{A}$ and $\mathrm{B}(\mathrm{P}<0.05)$.

Conclusions: For AMI patients after PCI, alprostadil combined with tanshinone IIa injection can effectively improve microcirculation and ventricular remodeling, improve cardiac function and reduce the occurrence of MACEs. This combination can be widely used in clinical practice.

Keywords: Alprostadil; tanshinone IIa injection; acute myocardial infarction (AMI); percutaneous coronary intervention (PCI); microcirculation; cardiac function; disease outcome

Submitted Sep 02, 2020. Accepted for publication Jan 02, 2021.

doi: 10.21037/apm-20-2147

View this article at: http://dx.doi.org/10.21037/apm-20-2147 


\section{Introduction}

Acute myocardial infarction (AMI) is common in clinical practice. When unstable atherosclerotic plaques rupture and hemorrhage, persistent spasm and acute thrombosis occur in the coronary arteries, leading to coronary artery occlusion and myocardial ischemia $(1,2)$. ECG, blood pressure and blood oxygen should be monitored immediately after AMI patients admission, and reperfusion therapy should be carried out as soon as possible to restore blood perfusion. PCI is recommended for patients who meet the class I recommendation within 12 hours of onset. Percutaneous coronary intervention (PCI) is the preferred treatment for AMI, as it is of great clinical value for patients to achieve rapid revascularization and rescue ischemic myocardial cells in the acute phase, but some patients still have poor prognosis. Approximately $25 \%$ of patients have the slow/no - reflow phenomenon after PCI, which can lead to cardiac insufficiency, myocardial ischemia, and left ventricular remodeling after AMI, significantly increasing the incidence of major adverse cardiac events (MACEs) (3). Therefore, it is necessary to treat these patients with drugs to improve their microcirculation and promote blood circulation to prevent blood stasis after PCI. AMI patients need antithrombotic therapy to improve microcirculation after PCI, including antiplatelet therapy, anticoagulant therapy and some other anti myocardial ischemia therapy, to reduce ventricular remodeling therapy. Alprostadil is an antithrombotic drug, tanshinone IIA is a traditional Chinese medicine preparation which can improve coronary circulation and inhibit thrombosis, Alprostadil is commonly used for treating peripheral arterial occlusive diseases because it can effectively dilate blood vessels, improve cardiac microcirculation, and reduce cardiac load (4). Tanshinone IIa injection has a good effect in the treatment of atherosclerosis. Therefore, this study investigated the effects of alprostadil combined with tanshinone IIa injection on microcirculation disorder, disease outcomes, and cardiac function in treating AMI patients after PCI to investigate their value in clinical treatment.

We present the following article in accordance with the CONSORT reporting checklist (available at http://dx.doi. org/10.21037/apm-20-2147).

\section{Methods}

\section{General information}

A total of 300 AMI patients who underwent PCI in our hospital between January 2013 and June 2018 were randomly selected. The patients were divided into group A, B and $\mathrm{C}$ by using the random number table method, with 100 patients in each group. There were 57 males and 48 females in the group A, with an average age of $58.13 \pm 5.16$ years (range: 47-76 years). Their underlying diseases included diabetes (28 cases), hypertension ( 45 cases), and dyslipidemia (42 cases). There were 64 males and 36 females in the group B, with an average age of $57.58 \pm 4.93$ years (range: 45-77 years). Their underlying diseases included diabetes (35 cases), hypertension (44 cases), and dyslipidemia (38 cases). There were 58 males and 42 females in the group C, with an average age of $58.06 \pm 4.84$ years (range, $47-78$ years). Their underlying diseases included diabetes (34 cases), hypertension (47 cases), and dyslipidemia (32 cases). There were no significant differences $(\mathrm{P}>0.05)$ in the general information (age and underlying diseases) between the three groups which were comparable. The study was conducted in accordance with the Declaration of Helsinki (as revised in 2013). The study was approved by institutional/regional/national ethics/committee/ethics board of Medical Ethics Committee of Hai'an People's Hospital (No.: HKL2020009) and informed consent was taken from all the patients.

\section{Inclusion and exclusion criteria}

Inclusion criteria: (I) all patients met the diagnostic criteria of AMI, and all underwent coronary angiography. (II) All patients were within $12 \mathrm{~h}$ after myocardial infarction (MI) and met the indications of PCI, and PCI was successful in all patients.

Exclusion criteria: (I) dysfunction of an organs such as the liver or kidney; (II) malignant tumors; (III) active visceral hemorrhage; (IV) recent history of trauma, surgery, or cerebral hemorrhage; (V) coagulation disorders; and (VI) neurological or mental disorders.

\section{Material methods}

All patients underwent $\mathrm{PCI}$ and were treated with medicines such as atorvastatin, clopidogrel, and aspirin. At the same time, the patients were also treated for other underlying diseases, such as hypertension, diabetes, and dyslipidemia. The group A received an intravenous infusion of $10 \mathrm{mg}$ of alprostadil (Hainan Bikai Pharmaceutical Co., Ltd., H2O103292) $30 \mathrm{~min}$ before the PCI, and the alprostadil treatment was continued for 7 days after surgery. The group 
B was treated with a tanshinone IIa injection (Shanghai No. 1 Biochemical \& Pharmaceutical Co., Ltd., H31022558) $30 \mathrm{~min}$ before PCI $(80 \mathrm{mg}$ added to $250 \mathrm{~mL}$ of $0.9 \%$ sodium chloride, intravenous infusion, qd), and the postoperative dose was the same as that before surgery (for 7 days). The group $\mathrm{C}$ was treated with tanshinone IIa injection and alprostadil. The treatment method and dose were the same as those in group A and group B.

\section{Observation indicators}

Comparison of cardiac function between the three groups Cardiography was performed after postoperative treatment by using color Doppler ultrasound. The frequency of 2.5 MHz was used. The left ventricular long axis view, left ventricle short axis view, and apical four-chamber view of the heart were routinely examined to detect the left ventricular end-diastolic diameter (LVEDD), enddiastolic left ventricular posterior wall thickness (LVPWD), left ventricular ejection fraction (LVEF), left ventricular end-systolic diameter (LVESD), interventricular septum thickness (IVST) and the ratio of the maximum blood flow of left ventricle in early diastolic phase and maximum atrial systolic blood flow (E/A ratio) in both groups.

\section{Myocardial contrast echocardiography (MCE) in three groups}

MCE was performed in three groups after postoperative treatment, and the analysis was performed off-line by using the MCE analysis software. The equation for calculation was $y=A\left(1-e^{-\beta t}\right)$, where A refers to myocardial blood volume, $\beta$ refers to the myocardial blood velocity, and $A \beta$ equals the local myocardial blood flow. The contralateral noninfarct area was selected as the control area. The A and $\beta$ values were measured, and the corrected values were obtained by comparing the values of the normal area and the lesioned area.

Comparison of postoperative perfusion in the three groups The thrombolysis in myocardial infarction (TIMI) myocardial perfusion grade (TMPG) results were used to evaluate postoperative cardiac perfusion. The TMPG has four grades. Grade 0 refers to no contrast medium entering the myocardium, no or little transient contrast myocardial contrast medium staining; grade 1 refers to extremely slowly wash-in, myocardial angiography showed ground glass opacification, and the retention time of the contrast agent is more than $30 \mathrm{~s}$; grade 2 refers to slow wash-in and delayed wash-out, myocardial angiography showed ground glass opacification, and the retention time of the contrast agent exceeds three cardiac cycles; grade 3 refers to a normal washin and normal wash-out, myocardial angiography showed ground glass opacification, and no retention of contrast agent. Grades $2-3$ indicate good myocardial perfusion, and grades $0-1$ indicate poor myocardial perfusion.

\section{Observation of MACEs}

MACEs were observed during hospitalization and the 12-month follow-up after the surgery, which including severe heart failure (HF), cardiac death, malignant arrhythmia, and recurrent MI.

\section{Statistical methods}

Data were analyzed with SPSS 19.0 software. Count data were expressed as percentages (\%), and the chi-squared test was used for intergroup comparisons. Measurement data were expressed as $\bar{x} \pm \mathrm{s}$. Analysis of variance was used to compare the date between three groups, the independentsample $t$-test were used to compare the data between every two groups. $\mathrm{P}<0.05$ was considered statistically significant.

\section{Results}

Comparison of cardiac function between the two groups

After treatment, the LVEDD, LVESD, LVPWD, IVST, LVEF, and the E/A ratio of the group $\mathrm{C}$ were all significantly better than those of the group A and group B $(\mathrm{P}<0.05)$ (Table 1).

\section{Comparison of the MCE results between the two groups}

The $A \beta$ of the group $C$ at 7 days after surgery was significantly higher than that of the group A and group B $(\mathrm{P}<0.05)$ (Table 2).

\section{TMPG grading of patients in the two groups after surgery}

Based on the TMPG classification, patients with grades 2-3 in the group $\mathrm{C}$ was significantly more common than those in the group $\mathrm{A}$ and group $\mathrm{B}(\mathrm{P}<0.05)($ Table 3$)$.

\section{Comparison of MACEs between the two groups}

The incidences of malignant arrhythmia, recurrent HF, 
Table 1 Comparison of cardiac function between the three groups $(\bar{x} \pm s)$

\begin{tabular}{|c|c|c|c|c|c|c|c|}
\hline Group & Case (n) & LVEDD & LVESD & LVPWD & LVEF (\%) & IVST & $E / A$ \\
\hline Group B & 100 & $46.6 \pm 5.7$ & $34.2 \pm 5.6$ & $9.9 \pm 0.8$ & $49.7 \pm 5.3$ & $9.8 \pm 0.9$ & $0.9 \pm 0.2$ \\
\hline Group C & 100 & $44.5 \pm 6.6$ & $31.5 \pm 4.8$ & $9.2 \pm 0.9$ & $56.1 \pm 4.6$ & $9.5 \pm 0.7$ & $1.1 \pm 0.4$ \\
\hline $\mathrm{F}$ & & 4.980 & 11.238 & 25.135 & 54.537 & 22.993 & 24.138 \\
\hline
\end{tabular}

LVEDD, left ventricular end-diastolic dimension; LVESD, left ventricular end-systolic dimension; LVPWD, left ventricular posterior wall thickness; LEVF, left ventricular ejection fraction; IVST, interventricular septum thickness.

Table 2 Quantitative detection of MCE in three groups of patients $(\bar{x} \pm s)$

\begin{tabular}{lcc}
\hline Group & Case $(\mathrm{n})$ & $\mathrm{A} \beta$ at $7 \mathrm{~d}$ after surgery \\
\hline Group A & 100 & $4.21 \pm 1.15$ \\
Group B & 100 & $4.09 \pm 1.32$ \\
Group C & 100 & $5.24 \pm 1.14$ \\
F & & 27.469 \\
P & & 0.000 \\
\hline
\end{tabular}

MCE, myocardial contrast echocardiography.

Table 3 TMPG classification of the three groups of patients (n, \%)

\begin{tabular}{lccc}
\hline Group & Case $(\mathrm{n})$ & Grade 0-1 & Grade 2-3 \\
\hline Group A & 100 & $27(27.00)$ & $73(73.00)$ \\
Group B & 100 & $24(24.00)$ & $76(76.00)$ \\
Group C & 100 & $9(11.00)$ & $91(91.00)$ \\
$\chi^{2}$ & & \multicolumn{2}{c}{11.630} \\
P & & \multicolumn{2}{c}{0.000} \\
\hline
\end{tabular}

recurrent MI, death, and other MACEs in the group $\mathrm{C}$ were significantly lower than those in the group A and group B $(\mathrm{P}<0.05)$ (Table 4).

\section{Discussion}

Coronary heart disease (CHD) is one of the most common cardiovascular diseases in the clinic, which mainly occurs in middle-aged and elderly populations. With the progression of CHD, myocardial ischemia and hypoxia become aggravated, and myocardial cell apoptosis may occur, leading to myocardial necrosis and AMI. Coronary intervention has high value in treating AMI, as it can significantly improve the survival rate of post-MI patients and improve their prognosis and quality of life. PCI can restore the normal blood flow of the blood vessels in the epicardium, but it increases the risk of thrombus detachment, which greatly increases the likelihood of embolization in the distal microcirculation. In this case, the myocardial tissue cannot achieve reperfusion, which can lead to poor myocardial microcirculation and the slow/no-reflow phenomenon and has a great impact on prognosis (5-7). Therefore, the use of drugs to improve microcirculation after PCI is of great clinical significance. This study investigated the effects of alprostadil combined with tanshinone IIa injection on AMI patients after PCI.

The no-reflow phenomenon is associated with myocardial microcirculation disorders; it may be associated with endothelial dysfunction, ischemia-reperfusion injury, distal embolization, and other mechanisms; and it is associated with slow coronary blood flow after PCI, which is due to the embolization of the small peripheral blood vessels in the coronary arteries caused by long-term atherosclerosis and plaque deposition $(8,9)$. Our study found that patients with TMPG grades $2-3$ in group C made up a significantly higher proportion than the group $\mathrm{A}$ and $\mathrm{B}(\mathrm{P}<0.05)$, indicating that alprostadil combined with tanshinone IIa injection can effectively improve microcirculation after PCI. Alprostadil is widely used in cardiovascular diseases and can dilate blood vessels. Especially alprostadil can effectively improve circulation of ischemic injury, increase the activities of $\mathrm{Na}^{+} / \mathrm{K}^{+}$-ATPases and the deformation of red blood cells, and significantly improve microcirculation disorders. Alprostadil can prevent the adhesion and aggregation of platelets, inhibit the adhesion and migration of neutrophils, promote angiogenesis, repair the vascular endothelium, and increase the stability of the lysosomal membrane (10). A study by 
Table 4 Comparison of adverse cardiac events between the two groups (n, \%)

\begin{tabular}{lcccccc}
\hline Group & Case $(\mathrm{n})$ & Malignant arrhythmia & Recurrent HF & Recurrent Ml & Death & Total incidence \\
\hline Group A & 100 & $3(0.3)$ & $5(0.5)$ & $9(0.9)$ & $2(0.2)$ & $19(19.00)$ \\
Group B & 100 & $4(0.4)$ & $7(0.7)$ & $4(0.4)$ & $0(0.0)$ & $15(15.00)$ \\
Group C & 100 & $3(0.3)$ & $3(0.3)$ & $1(0.1)$ & $0(0.0)$ & $7(7.00)$ \\
$\chi^{2}$ & & & & 6.330 \\
P & & & & & 0.042 \\
\hline
\end{tabular}

Wei et al. (11) showed that the use of alprostadil after PCI can significantly improve the corrected TIMI frame count. Tanshinone IIa injection is a preparation of active substance obtained by sulfonation of diterpenoid quinone tanshinone IIa from Salvia miltiorrhiza, the main component is sodium tanshinone IIa sulfonate. Tanshinone IIA injection can significantly dilate coronary artery, increase coronary blood flow, improve myocardial metabolic disorder caused by hypoxia, so as to improve the ability of myocardial hypoxia tolerance. Tanshinone IIA can also improve blood microcirculation, increase the number of capillary network opening, speed up the blood flow rate, thus improving microcirculation disorder. Tanshinone IIA can inhibit ADP induced platelet aggregation, inhibit the synthesis and release of thromboxane $\mathrm{A} 2$ and promote the degradation of fibrin. It can reduce the whole blood and plasma viscosity of patients with coronary heart disease, cerebral ischemic stroke and myocardial infarction, reduce the hematocrit, and restore the hemorheological indexes of patients with anticoagulation and viscosity reduction. Tanshinone IIA can directly activate the calcium-activated potassium channels of smooth muscle cells in the coronary arteries, reduce the spontaneous electrical activity of vascular smooth muscle, and relax the coronary arteries, thus effectively improving the myocardial collateral circulation and local blood supply in the ischemic area. By these effects, alprostadil combined with tanshinone IIa injection improved microcirculation in AMI patients after PCI $(12,13)$.

With the occurrence of myocardial ischemia, the activity of myocardial cells decreased, ventricular remodeling may occur in patients with AMI, resulting in left ventricular enlargement and deformation, thereby reducing systolic function. Ventricular remodeling is associated with severe complications, such as HF and cardiac rupture, and has a significant impact on prognosis. MCE is a commonly used examination in clinical practice that can effectively evaluate the activity of cardiomyocytes with high specificity and sensitivity. In the early stage of reperfusion therapy, MCE can sensitively detect the changes in microcirculation perfusion. In this study, MCE at 7 days after PCI showed that $\mathrm{A} \beta$ in the observation group was significantly higher than that in the control group $(\mathrm{P}<0.05)$. The indexes of ventricular remodeling and cardiac function showed that the LVEDD, LVESD, LVPWD, IVST, LVEF, and E/A ratio in the group $\mathrm{C}$ after treatment were all significantly better than those in the group A and group $\mathrm{B}(\mathrm{P}<0.05)$, indicating that the alprostadil combined with tanshinone IIa injection after PCI can reduce ventricular remodeling, improve myocardial activity and effectively improve cardiac function. Tanshinone II a can improve blood microcirculation, promote the recovery of blood perfusion, reduce myocardial injury caused by hypoxia, improve myocardial contractility, promote myocardial regeneration and repair myocardial cells by dilating blood vessels. Tanshinone II a can also protect erythrocyte membrane and reduce infarct area of myocardial infarction. In the study by Dodson et al. (14), the combination of tanshinone IIa injection with PCI significantly improved cardiac function in patients. It may be that the combination of these two can promote neovascularization in ischemic areas, promote antimyocardial-ischemic and antioxidative effects, and improve ventricular remodeling. In this study, the incidences of malignant arrhythmia, recurrent HF, recurrent MI, death, and other MACEs in the observation group were significantly lower than those in the control group $(\mathrm{P}<0.05)$, indicating that alprostadil combined with tanshinone IIa injection can effectively improve the cardiac function of patients after PCI, improve blood perfusion of the heart, and reduce the occurrence of MACEs.

In summary, alprostadil combined with tanshinone IIa injection can effectively improve microcirculation in AMI patients after PCI, improve ventricular remodeling, improve cardiac function, and reduce the occurrence of MACEs. Therefore, it can be widely used in clinical practice. 


\section{Acknowledgments}

Funding: None.

\section{Footnote}

Reporting Checklist: The authors have completed the CONSORT reporting checklist. Available at http://dx.doi. org/10.21037/apm-20-2147

Data Sharing Statement: Available at http://dx.doi. org/10.21037/apm-20-2147

Conflicts of Interest: All authors have completed the ICMJE uniform disclosure form (available at http://dx.doi. org/10.21037/apm-20-2147). The authors have no conflicts of interest to declare.

Ethical Statement: The authors are accountable for all aspects of the work in ensuring that questions related to the accuracy or integrity of any part of the work are appropriately investigated and resolved. The study was conducted in accordance with the Declaration of Helsinki (as revised in 2013). The study was approved by institutional/ regional/national ethics/committee/ethics board of Medical Ethics Committee of Hai'an People's Hospital (No.: HKL2020009) and informed consent was taken from all the patients.

Open Access Statement: This is an Open Access article distributed in accordance with the Creative Commons Attribution-NonCommercial-NoDerivs 4.0 International License (CC BY-NC-ND 4.0), which permits the noncommercial replication and distribution of the article with the strict proviso that no changes or edits are made and the original work is properly cited (including links to both the formal publication through the relevant DOI and the license). See: https://creativecommons.org/licenses/by-nc-nd/4.0/.

\section{References}

1. Levine GN, Bates ER, Bittl JA, et al. 2016 ACC/ AHA Guideline Focused Update on Duration of Dual Antiplatelet Therapy in Patients With Coronary Artery Disease: A Report of the American College of Cardiology/ American Heart Association Task Force on Clinical Practice Guidelines. J Am Coll Cardiol 2016;68:1082-115.

2. He XM, Chen L, Luo JB, et al. Effects of rhBNP after
PCI on non-invasive hemodynamic in acute myocardial infarction patients with left heart failure. Asian Pac J Trop Med 2016;9:791-5.

3. Tanaka A, Kawarabayashi T, Nishibori Y, et al. No-reflow phenomenon and lesion morphology in patients with acute myocardial infarction. Circulation 2002;105:2148-52.

4. Wei W, Lai SC, Xie Y, et al. The Protective Effect of Target Temperature Management Combined with Prostaglandin $\mathrm{E}_{1}$ on Ischemia/Reperfusion Injury of Cerebral Micro-vascular Endothelium of ROSC Rat. Sichuan Da Xue Xue Bao Yi Xue Ban 2016;47:310-5.

5. Niccoli G, Scalone G, Lerman A, et al. Coronary microvascular obstruction in acute myocardial infarction. Eur Heart J 2016;37:1024-33.

6. Süleymanoğlu M, Rencüzoğulları İ, Karabağ Y, et al. The relationship between atherogenic index of plasma and no-reflow in patients with acute ST-segment elevation myocardial infarction who underwent primary percutaneous coronary intervention. Int J Cardiovasc Imaging 2020;36:789-96.

7. A X, Li Z, Luo W, et al. Long-term compound danshen dripping pills therapy reduces the no-reflow phenomenon in nondiabetes mellitus patients after primary percutaneous coronary intervention for acute myocardial infarction. Ann Palliat Med 2020;9:1144-51.

8. Kloner RA. No-reflow phenomenon: maintaining vascular integrity. J Cardiovasc Pharmacol Ther 2011;16:244-50.

9. A X, Li Z, Luo W, Chai J. Long-term compound danshen dripping pills therapy reduces the no-reflow phenomenon in nondiabetes mellitus patients after primary percutaneous coronary intervention for acute myocardial infarction. Ann Palliat Med 2020;9:1144-51.

10. Lawall H, Pokrovsky A, Checinski P, et al. Efficacy and Safety of Alprostadil in Patients with Peripheral Arterial Occlusive Disease Fontaine Stage IV: Results of a Placebo Controlled Randomised Multicentre Trial (ESPECIAL). Eur J Vasc Endovasc Surg 2017;53:559-66.

11. Wei LY, Fu XH, Li W, et al. Effect of Intravenous Administration of Liposomal Prostaglandin E1 on Microcirculation in Patients with ST Elevation Myocardial Infarction Undergoing Primary Percutaneous Intervention. Chin Med J (Engl) 2015;128:1147-50.

12. Li ZM, Xu SW, Liu PQ. Salvia miltiorrhizaBurge (Danshen): a golden herbal medicine in cardiovascular therapeutics. Acta Pharmacol Sin 2018;39:802-24.

13. Wei B, Li WW, Ji J, et al. The cardioprotective effect of sodium tanshinone IIA sulfonate and the optimizing of 
therapeutic time window in myocardial ischemia/reperfusion injury in rats. Atherosclerosis 2014;235:318-27.

14. Dodson TB, Cheng JT, Zhu MJ, et al. Tanshinone II a joint yiqi compound injection pulse injection the effect of

Cite this article as: Lu Y, Yan Y, Liu X. Effects of alprostadil combined with tanshinone IIa injection on microcirculation disorder, outcomes, and cardiac function in AMI patients after PCI. Ann Palliat Med 2021;10(1):97-103. doi: 10.21037/apm-202147
PCI postoperative heart function in patients with acute myocardial infarction. Journal of Chinese Traditional Medicine 2017;032:1886-8. 